\title{
Membranes as Biomaterials
}

\author{
Yoshito IKADA \\ Research Center for Biomedical Engineering, Kyoto University, \\ 53 Kawahara-cho, Shogoin, Sakyo-ku, Kyoto 606, Japan
}

(Received November 26, 1990)

\begin{abstract}
Five different artificial organs or tissues are selected from our studies to show how membranes are used as biomaterials. They are artificial kidney, artificial lung, artificial skin, artificial pancreas, and artificial cornea. The membranes in these artificial organs are mostly employed to allow the selective permeation of some substances, similar to those in industrial applications. However, biocompatibility is additionally required in the medical applications of these membranes. It is shown that coupling of poly(ethylene glycol) onto the cellulose membrane used for hemodialysis greatly improves the blood compatibility, while graft polymerization of acrylamide onto a silicone membrane used for extracorporeal membrane oxygenation largely reduces clot formation on the surface. The silicone membrane is shown to be effective also for protection of damaged skin, when used with a porous collagen sheet. It is further demonstrated that poly(vinyl alcohol) membranes without and with immobilized collagen can be used for the entrapment of Langerhans islets and for the repair of damaged corneal tissue, respectively. KEY WORDS Artificial Organs / Hemodialysis Membrane / Extracorporeal Membrane 0xygenation / Biocompatibility / Surface Grafting
\end{abstract}

Introduction

If a membrane is defined as the two-dimensional material through which some substances can selectively permeate, our body comprises many kinds of membrane such as cell membrane, basement membrane, peritoneal membrane, and skin. Therefore, it seems very reasonable to suppose that a large number of artificial membranes must have been already applied as biomaterials in medical fields on a large scale. However, this is not always true. For instance, artificial lipid membranes have been very extensively studied as liposome for drug delivery systems over last two decades, but not yet clinically applied. It should be kept in mind that there are many problems in applying the artificial materials to medicine. One of them is the poor biocompatibility of existing materials. Very often, the technical term "biocompatibility"1) is used 


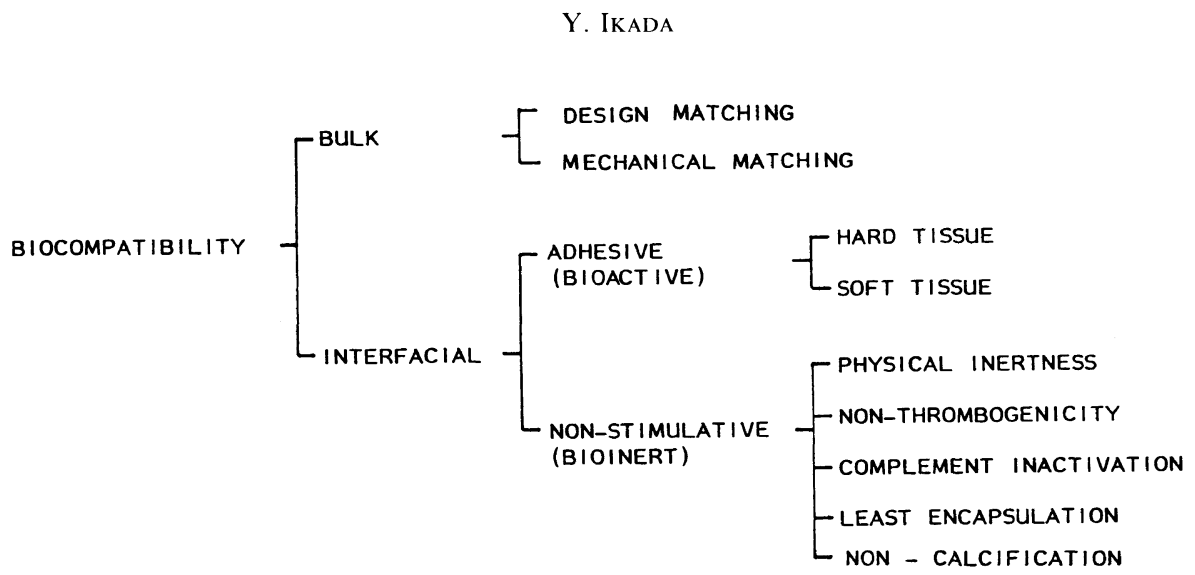

Figure 1. Classification of biocomopatibility.

synonymously with "non-toxicity", but, in this presentation, they are regarded as different concepts. Fig.1 demonstrates what the biocompatibility is. As can be seen, it can be divided into two large groups; bulk and interfacial biocompatibility.

This presentation focuses on the improvement of biocompatibility of biomedical membranes to be used as the most important parts of artificial organs or tissues. They cover artificial kidney, artificial lung, artificial skin, artificial pancreas, and artificial cornea. All of the experimental results described below were obtained in our laboratory in collaborative works with medical doctors.

1. Artificial Kidney

The main part of the currently used artificial kidneys is the semipermeable polymeric membrane set-up in hollow fiber-type hemodialyzers. The total surface area in one hemodialyzer ranges from 0.5 to $2.0 \mathrm{~m}^{2}$. A biggest topic of the hemodialysis membrane is removal of ${ }^{B}$-microglobulin which is thought to be a pathogen of amyloidosis observed in long-term hemodialysis cases. This protein with the molecular weight of 11,800 can be removed from blood by the so-called high performance membranes having relatively large pore size. They are widely used in clinics to remove $\beta_{2}$-microglobulin at present.

Among the problems of hemodialysis membranes to be solved is the lack of blood compatibility. Especially, the cellulosic membranes, currently employed on the largest scale in hemodialysis, are known to activate most strongly the complement system which plays an important role in the initial stage of self-defense of body against foreign-body invasion. The transient leucopenia appearing most remarkably about half an hour after the start of hemodialysis treatment seems to occur as a result of complement activation ${ }^{2}$. In an attempt to reduce the complement activation, we modified the cellulose surface by a polymer coupling technique without altering the permeation properties of the membrane. The polymer employed for the surface modification is poly(ethylene glycol)(PEG) with a terminal carboxyl group. Through esterification between the carboxyl group and the hydroxyl group on the 
cellulose surface, the PEG chain was grafted on the membrane surface to prevent the direct interaction of complement proteins with the membrane, which would otherwise induce the transient leucopenia and other side effects.

Fig. 2 shows the percent get of CH50 decrease which is a measure of complement activation ${ }^{3)}$. Apparently, CH50 greatly decreases by grafting of PEG chains, especially when PEG with low molecular weights such as 400 and 1,000 are employed for the surface modification. It appears that the direct interaction between cellulose and plasma proteins is reduced to a higher degree as the density and the length of the PEG chains become higher and longer, respectively.

As good results were obtained for the surface-modified membrane in animal experiments, the polymer coupling reaction was performed on a plant scale. After trials for a few years, industrialization of the new membrane became successful. Fig.3 shows the dependence of leucocyte number on the amount of the grafted chains during hemodialysis of patients ${ }^{4)}$. As can be seen, the surface modification of membrane by the polymer coupling greatly reduces the protein adsorption on the membrane also in vivo and becomes virtually zero when the graft amount approaches $200 \mu \mathrm{g} / \mathrm{g}$.

The remarkable reduction in protein adsorption onto the membrane during hemodialysis implies that thrombus formation on the membrane surface will be also suppressed by the polymer coupling. Indeed, scanning electron microscopic observation on the membrane surface after hemodialysis of patients indicated the greatly reduced thrombus formation, in comparison with that of unmodified cellulose membrane. Thus, it is expected that the concentration of heparin to be administered during hemodialysis will be largely reduced.

\section{Artificial Lung}

The artificial lung is a medical device to be applied to patients with diseases in lung for $\mathrm{CO}_{2}$ removal from blood and $\mathrm{O}_{2}$ supply to blood of the patients. The gas exchange using membrane-type artificial lungs is called extracorporeal membrane oxygenation(ECMO). Two types of membrane are currently employed for ECMO; porous and non-porous. The material of porous membranes is mostly polypropylene and the porous structure is created by fibrilization occurring as a result of chain extension. The gas exchange takes place through the micropores and hence is very high in gas permeation rate. However, this type of membrane has a very short life time, because serum leakage occurs through the pores after protein adsorption. On the other hand, the nonporous membrane is made of silicone which has the highest gas permeability among the conventional polymers. Although the silicone membrane does not have such a problem as serum leakage because of its non-porous structure, its life time is also not long enough. The reason is poor blood compatibility of the silicone surface. Similar to hemodialysis, ECMO is conducted with the use of heparin to prevent blood coagulation during the treatment, but the heparin concentration is much higher at the ECMO treatment than the hemodialysis because of much 


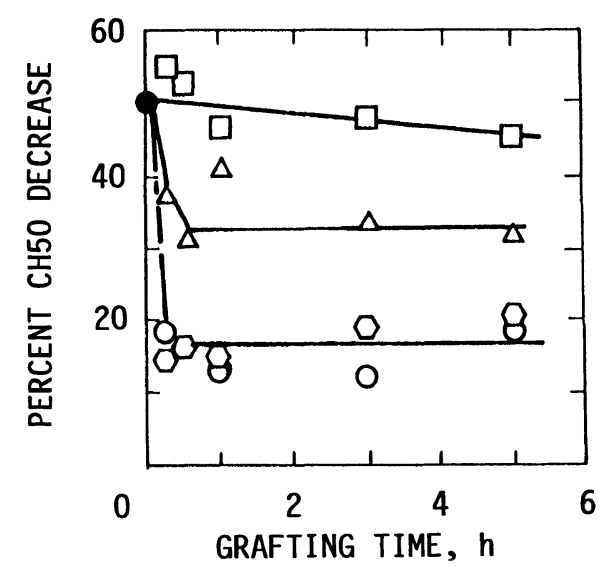

Figure 2. Effect of the PEG grafting time on the activation decrease for the cellulose hollow fiber.

( $\square$ ) Mw $8000,(\triangle)$ Mw 4000, ( 0 ) Mw 1000,

( $\bigcirc$ ) Mw 400

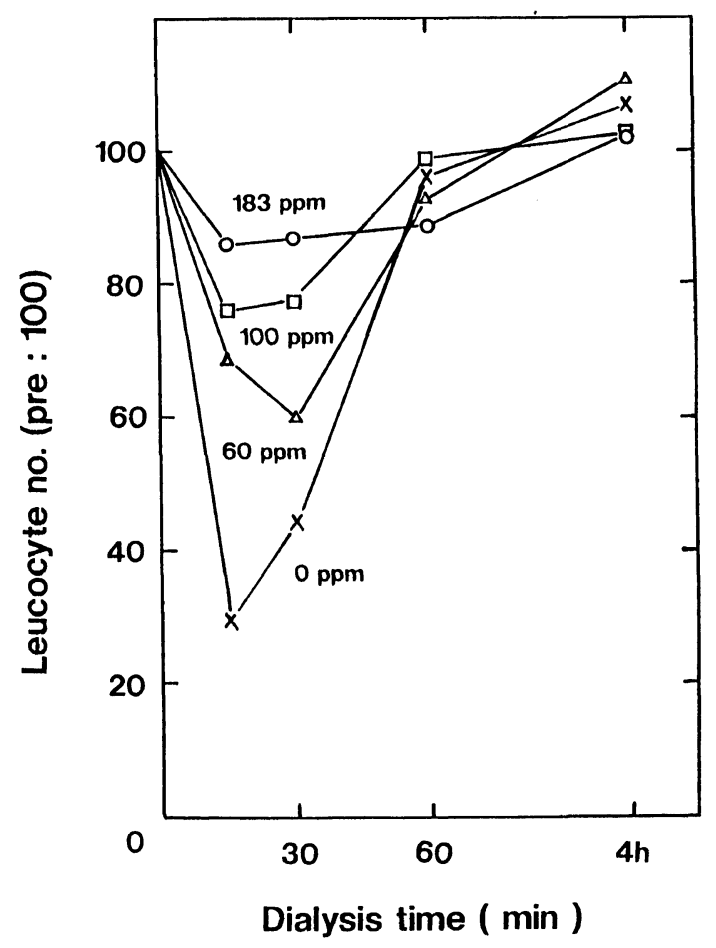

Figure 3. Change of the leucocyte number during dialysis with the cellulose membrane grafted with poly(ethylene glycol) to different surface concentrations. 

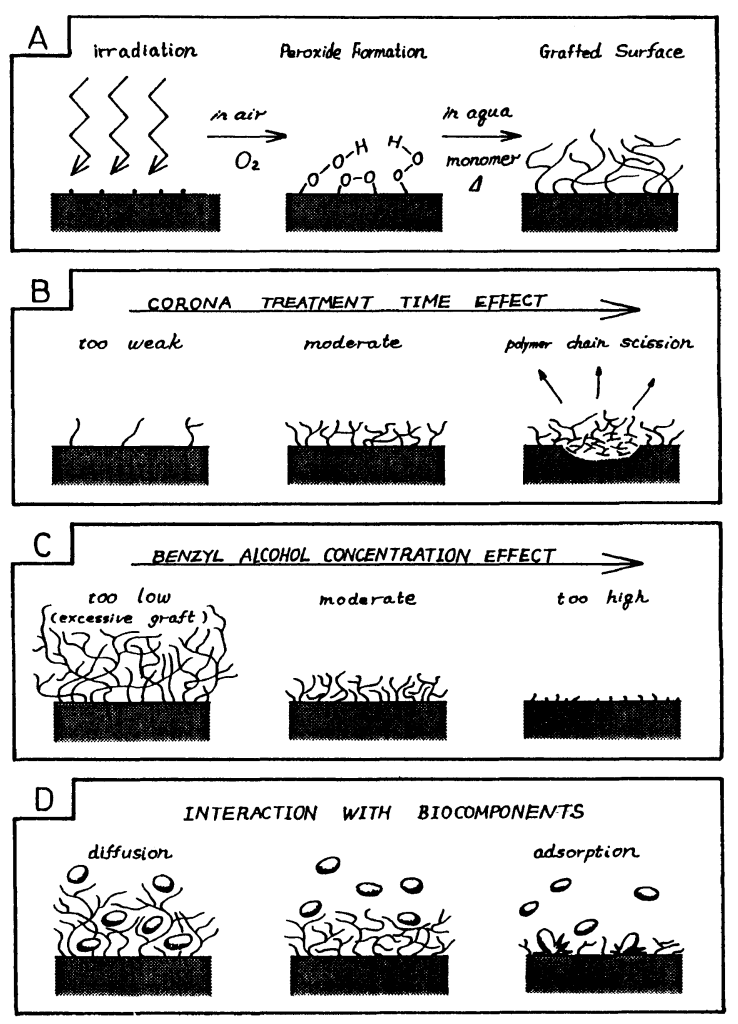

Figure 4. Schematic representation of surface graft polymerization and the protein interaction with the grafted surfaces.

higher blood flow. In addition, ECMO is usually carried out for a much longer time than hemodialysis. Therefore, excellent blood compatibility is more strongly required for the membrane of ECMO than that of hemodialysis .

As it seems easier for the non-porous membrane to improve the blood compatibility than the porous one, we have attempted the surface modification of silicone hollow fibers to give good blood compatibility. In the case of silicone, we cannot use the polymer coupling technique which was applied to the cellulose modification, because silicone itself has no functional groups for the polymer coupling. Therefore, we employed the graft polymerization technique for the silicone membrane. Fig. 4 gives the schematic representation of the surface modification by the graft polymerization. First, the substrate polymer is exposed to oxidation media to generate polymer peroxides on the surface layer. Utilizing the peroxides as polymerization initiator, one can perform the graft polymerization of any monomers polymerizable through the radical mechanism. Among the oxidation media are high-energy radiation ${ }^{\text {) }}$, lowtemperature plasmas through glow ${ }^{6)}$ and corona discharge ${ }^{7)}$, ultraviolet radiation ${ }^{8)}$, and ozone gas 9 ) 

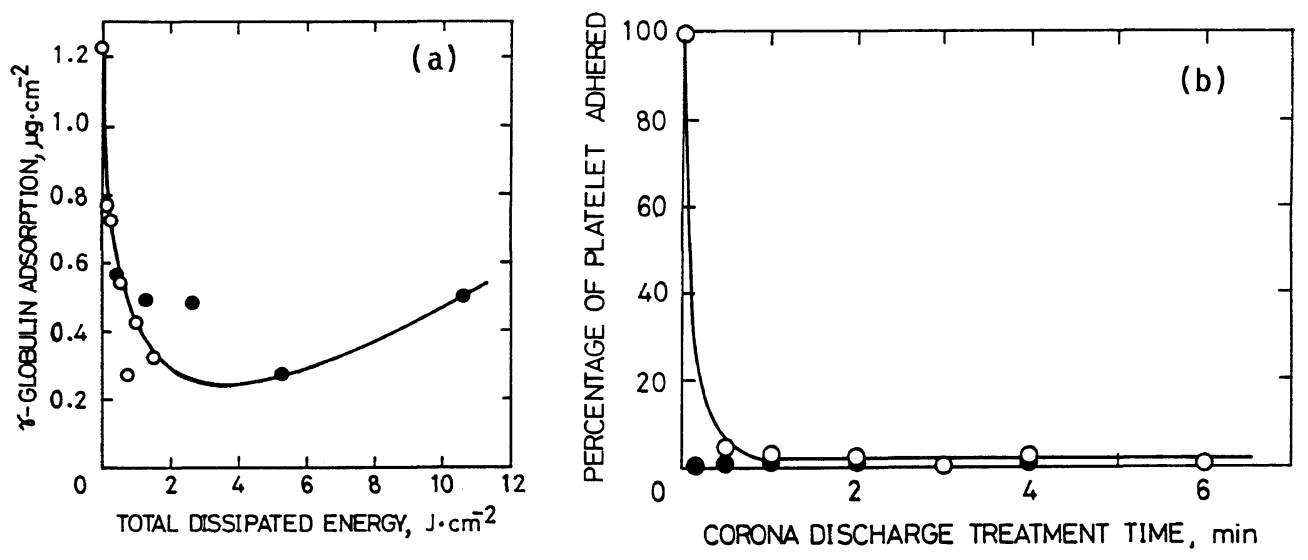

Figure 5. $\gamma$-Globulin (a) and Platelet adhesion (b) to poly(dimethyl acrylamide)-grafted silicones .

(O) $6 \mathrm{kV}$ corona treatment

( ) $15 \mathrm{kV}$ corona treatment

The result of graft polymerization of acrylamide onto the silicone surface is shown in Fig. ${ }^{10)}$. As is apparent, the graft polymerization significantly reduces the protein adsorption and the platelet adhesion. In vivo evaluation inserting the surface-modified silicone hollow fiber into an artery of dog revealed that only little thrombus formation was observed on the grafted silicone surface, compared with the unmodified surface, where severe clotting was recognized ${ }^{11)}$. An oxygen permeation experiment on the modified silicone showed that the boundary layer resistance which is a governing factor of the membrane permeation efficacy decreased to an appreciable extent by the surface graft polymerization ${ }^{12)}$.

\section{Artificial Skin}

Our skin is the largest among the living organs, breathing and protecting our body. When the skin is damaged to a wide area, water dissipation from the body and the bacterial invasion into the body should be prevented first of all. For this purpose, wound covers as well as artificial skins are clinically employed at present. The wound covers are generally used to protect the damaged skin from the outer environment, mostly acting as a simple mechanical barrier, while the term "artificial skin" is of ten used when the regeneration of new skin tissue is promoted by the biomaterial. The materials used for wound covering and the tissue regeneration include silicone, polyurethane, hydrogels, regenerated collagen, modified porcine skin, and regenerated chitin.

The artificial skins which are currently most extensively studied are composed of regenerated collagen with or without seeded skin cells. Mostly, a porous collagen sheet is used because it provides a very good 
Table 1 Difference in preparing method between our "artificial skin" and Stage 1 membrane by Yannas et al.

\begin{tabular}{lll}
\hline & Our "artificial skin" & Stage I membrane \\
\hline Collagen materials & Soluble pig skin collagen & Insoluble bovine hide collagen \\
$\begin{array}{l}\text { Production of sponge } \\
\text { structure }\end{array}$ & $\begin{array}{l}\text { Mixed solution of collagen and } \\
\text { GAG is directly freezed and } \\
\text { freeze-dried }\end{array}$ & $\begin{array}{l}\text { Collagen and GAG are co- } \\
\text { precipitated and the precipitate } \\
\text { is freeze-dried }\end{array}$ \\
\hline
\end{tabular}

substrate for cell ingrowth, as collagen has ligands for the receptor of fibroblast. Yannas et al. have first demonstrated that a porous collagen-glycosaminoglycan composite sheet lined with a thin silicone membrane is very effective in regeneration of deeply damaged skins ${ }^{13)}$.

We have also prepared a similar bilayered artificial skin from type-I collagen and silicone, but without adding any glycosaminoglycan to collagen. The gas permeability of the silicone membrane is given in Table 1, together with other properties ${ }^{14)}$. The most unique feature of this collagen membrane is to disappear as a result of bioabsorption. In other words, this artificial membrane provides a scaffold for the cells to reconstruct a new living tissue membrane, concurrently being biodegraded by the enzymatic action. The results of clinical trials are promising until now, although weak infection was observed in some cases. Such a biodegradable membrane acting as a temporary scaffold seems to be a good target for the future study on biomedical materials.

\section{Artificial Pancreas}

Pancreas is the metabolic internal organ which secretes insulin and glucagon in response to the glucose concentration in blood. When the pancreas is severely and irreversibly damaged, the patient needs the insulin supply from outside or inside. Currently, insulin is supplied to patients from outside by intravenous injection. As this administration method is associated with several problems, two kinds of artificial pancreas are being studied at present. One is the mechanical artificial pancreas composed of a glucose sensor, an insulin injection pump, and a controlling unit. The other is called biological artificial pancrease, because it is a hybrid type composed of Langerhans islets and an artificial membrane. Since the latter device is used entirely implanted in body, the Langerhans islets collected from animals of other spieces should be completely isolated in body. Otherwise, the implanted Langerhans islets would be severely attacked by the host immune system. To avoid this host self-dense attack, the Langerhans islets should be isolated, for instance, by inclusion in minibag, microcapsule, or hollow fiber.

We are entrapping rat Langerhans islets in a small tube made of poly(vinyl alcohol)(PVA) hydrogel membrane. The diameter and length of the tube are 2 and $40 \mathrm{~mm}$, respectively. The membrane has such a high water content as $97 \mathrm{wt} \%$ to allow the insulin transport through the 


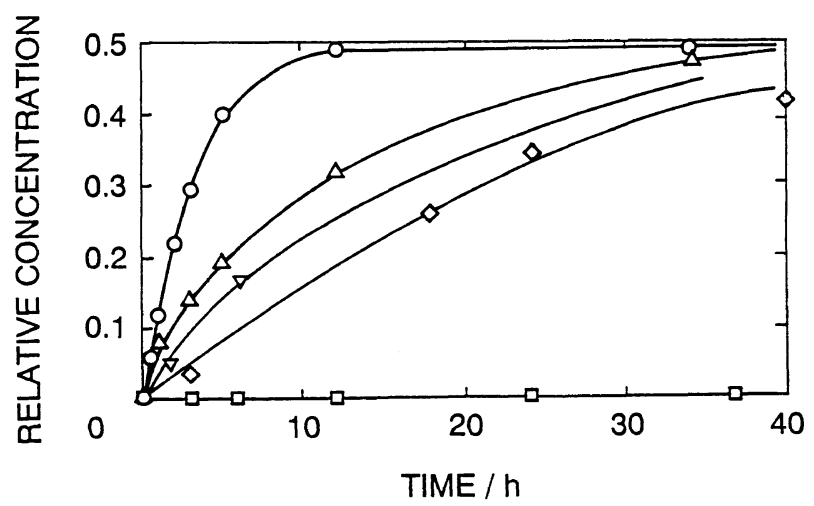

Figure 6. Permeability of biological substances through PVA hydrogel membrane. (water content $=97 \%$, surface area $=0.64 \mathrm{~cm}^{2}$ )

(O) glucose $(\mathrm{Mw}=180 ;$ conc. $=100 \mathrm{mg} / \mathrm{dl}),(\Delta)$ insulin $(\mathrm{Mw}=5,700 ;$ conc. $=250 \mu \mathrm{U} / \mathrm{ml})$, $(\nabla)$ heparin $(\mathrm{Mw}=20,000 ;$ conc. $=1000 \mathrm{U} / \mathrm{ml}),(\diamond) \mathrm{BSA} \quad(\mathrm{Mw}=67,000 ;$ conc. $=4000 \mathrm{mg} / \mathrm{dl})$, (ם) $\operatorname{IgG}(\mathrm{Mw}=180,000 ;$ conc. $=1500 \mathrm{mg} / \mathrm{dl})$

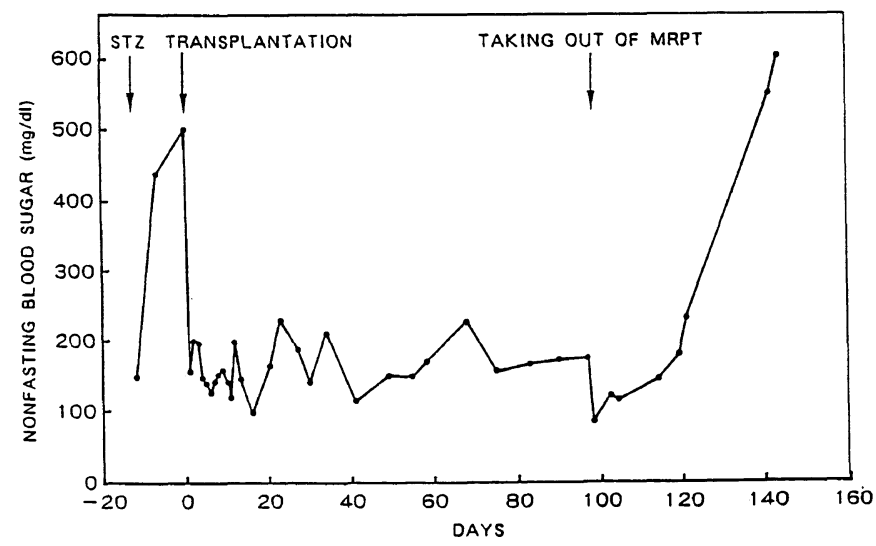

Figure 7. Charge of the rat blood sugar concentration by transplantation of rat Langerhans islets entrapped in a poly(vinyl alcohol) hydrogel membrane.

membrane. Immunoglobulins such as IgG cannot permeate through the water-swollen hydroge1, as shown in Fig. ${ }^{15)}$. Thus, this hydrogel membrane not only allows the transport of cell products and nutrients, but also protects the islet cells from the host attacks through the immunoglobulins and immunocytes. Another important requisite of the membrane for the artificial pancreas is the minimal encapsulation by collagenous tissues when implanted in body. Our PVA hydrogel was found to be hardly encapsulated with the tissue in contrast with silicone and polytetrafluoroethylene when implanted in peritoneal cavity of rat. Encouraged by these results, we implanted the hydrogel membrane tube entrapping rat Langerhans islets in another rat peritonea. Fig. 7 shows one of the results ${ }^{16)}$. It is seen that the blood glucose concentration of the diabetic rat was maintained at the normal level so far as the 
artificial pancreas was present in the rat.

\section{Artificial Cornea}

When the corneal tissue of eye is partially or totally damaged to a significant extent, corneal transplantation is performed, but the number of donor eyes is quite limited. Therefore, a number of studies have been carried out to develop artificial corneas from biomaterials, for instance, poly(methyl methacrylate) and polysulfone, but most of the trials have failed in developing such artificial corneas that can be clinically used for a long period of time with high reliability.

We have investigated two types of artificial cornea, as shown in Fig.8. Both of them are made of the PVA hydrogel through which water, oxygen, and other nutrients for corneal cells can permeate. Another important requirement for the biomaterial to be used as the artificial cornea is the microscopic bioadhesion to the surrounding corneal tissue. As the PVA hydrogel with a high water content is the least bioadhesive among polymeric biomaterials, similar to other hydrogels, we modified the surface of PVA hydrogel membrane so as to become bioadhesive by grafting of collagen molecules which are known to be the best promoter of bioadhesion. Indeed, many fibroblasts attached and proliferated well on the collagen-immobilized PVA membrane ${ }^{17)}$. Fig.9 shows the result of the in vitro study.

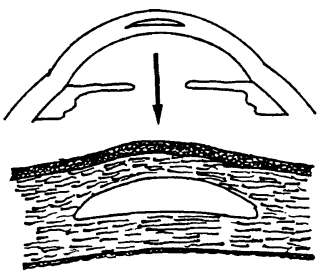

(a)

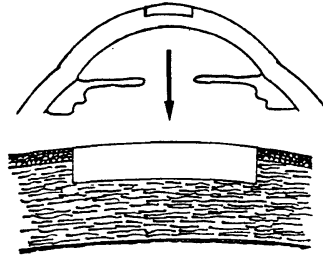

(b)
Figure 8. Schematic representation of two kinds of artificial cornea.

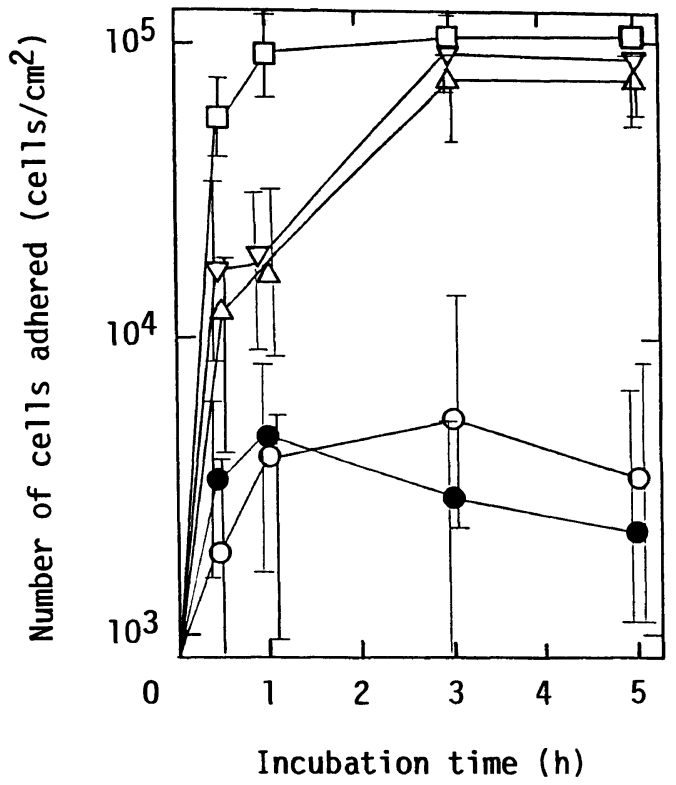

Figure 9. Corneal epithelial cell adhesion on varlous PVA gel surfaces in the presence of $10 \%$ FCS. PVA (O), PVA-HMDI ( $)$, PVA-HMDI-collagen ( $\square$ ), PVA-HMDI-fibronectin $(\nabla)$, and PVA-HMDI-RGDS $(\Delta)$ were subjected to the experiment. (HMDI: hexamethylene di isocyanate) 
When the collagen-immobilized membrane was implanted in the rabbit cornea as shown in Fig.8(a), the corneal tissue surrounding the implant at the outer side became thinner with the implantation time and was finally rejected. A plausible reason for the tissue thinning is insufficient supply of the nutrients to the corneal tissue, in other words, prevention of the free transport of the cell nutrients. Also, implantation of the hydrogel as shown in Fig.8(b) gave no good bioadhesion, although the membrane surface was covalently covered with collagen. Probably, improper fixation of the hydrogel membrane to the corneal tissue with suturing may be the main reason for the failure.

\section{REFERENCES}

1. "Definitions in Biomaterials, D.F. Williams ed., Elsevier, Amsterdam, 1987.

2. P.R. Craddock, J. Fehr, A.P. Dalmasso, K.L. Brigham, and H.S. Jacob, J. Clin. Invest., $\underline{59}, 879$ (1977).

3. E. Corretge, A. Kishida, H. Konishi, and Y. Ikada, Polymers in Medicine III, C. Migliaresi et al. ed., Elsevier, Amsterdam, 1988, p.61.

4. T. Akizawa, K. Kino, S. Koshikawa, Y. Ikada, A. Kishida, M. Yamashita, and K. Imamura, Amer. Soc. Artif. Internal Org. Trans., $\underline{35}, 333$ (1989).

5. M. Suzuki, Y. Tamada, H. Iwata, and Y. Ikada, Physicochem. Aspects Polym. Surfaces, 2, K.L. Mittal ed., Plenum Press, New York, 1983, p.923.

6. M. Suzuki, A. Kishida, H. Iwata, and Y. Ikada, Macromolecules, $\underline{19}$, $1804(1986)$.

7. H. Iwata, A. Kishida, M. Suzuki, Y. Hata, and Y. Ikada, J. Polym. Sci., Polym. Chem., 26, 3309 (1988).

8. Y. Uyama and Y. Ikada, J. Appl. Polym. Sci., 36, 1087 (1988).

9. Y. Takebayashi, K. Fujimoto, and Y. Ikada, 35 th Annual Kobe Meeting of Polymer Science, Abst. 35, 9 (1989).

10. A. Kishida, Y. Hata, and Y. Ikada, Polymer Preprints Japan, $\underline{36}, 831$ (1987).

11. H. Tadokoro, K. Fujimoto, and Y. Ikada, unpublished result.

12. A. Kishida, Y. Ikada, T. Higashimura, and T. Masuda, Polymer Preprints Japan, 35, 501 (1986).

13. I.V. Yannas and J.F. Burke, J. Biomed. Mater. Res., 14, 65 (1980).

14. S. Suzuki, K. Matsuda, N. Isshiki, Y. Tamada, K. Yoshioka, and Y. Ikada, Br. J. Plas. Surg., 43,47 (1990).

15. T. Fujisato, T. Okada, Y. Tabata, and Y. Ikada, Preprints of Japanese Society for Biomaterials, 11, 90 (1989).

16. K. Inoue, S. Sumi, Gu Y-J, T. Fujisato, S. Higashide, H. Minote, K. Takaori, M. Yun, K. Uchida, T. Tobe, Y. Tabata, and Y. Ikada, HPB Surgery, 2, suppl., 25 (1990).

17. H. Kobayashi and Y. Ikada, Current Eye Research, submitted. 